$\Phi=$ 줄

\title{
Rehydration of air dried oral smears using honey
}

\author{
Subashini.V*, Pratibha Ramani, Mpbrundha, Anuja. N, Herald J Sherlin, \\ Gheena .S Abhilasha R, Giffrina Jayaraj \\ Department of Oral and Maxillofacial Pathology, Saveetha Dental College and Hospital, Chennai 600077, India \\ *Corresponding author E-mail: shini2810@gmail.com
}

\begin{abstract}
Background: Papanicolaou stains, very reliable cytological staining in diagnosis of oral carcinoma. In routine practice, $95 \%$ ethanol is used to fix the slides while preparing for Pap smears. The problems associated with pap smear such as short of supply and storage of alcohol, wet fixing of slides and transporting them to the cytology centre if the Pap smears are prepared at a peripheral centre. If an alternative method of rehydrating the air-dried oral smears is known we can overcome these problems. Until the date only few studies regarding rehydration of oral smears as a substitute for wet fixation.

Aim: The aim of this study is to use honey as an alternative for rehydrating air dried oral smears.

Material and methods: This study was done over than 3 months. Two sets of oral smears wet and air dried ,Overall 40 smears were examined; The first set were fixed in $95 \%$ ethyl alcohol. The other set of smears were air dried and rehydrated with honey solution(2 parts honey and 1 part saline)Both the smears were stained with Pap stain, examined for preservation of cytomorphological features and evaluated by two experienced pathologists using the light microscope.

Results and analysis: This study revealed that immersion of air dried PAP smears in honey for 30 minutes is effectively same as wet smear which is routine method

Conclusion: Rehydration, followed by fixation of air-dried smears, is a simple, feasible, applicable and reliable fixation technique, which is comparable to the wet-fixed conventional technique, used for oral smears and can be applied for evaluation on a routine basis.
\end{abstract}

\section{Introduction}

PAP smear is worldwide used as a useful method for early detection of cancerous lesions. Smears are collected by clinicians or paramedical staff in clinics, hospitals or health centres. The most common one's fixative used in exfoliative cytology is $95 \%$ ethylalcohol. It gives excellent nuclear and cytoplasmic morphology, so used as a standard fixative. When slides need to be transported from clinic to lab, sometimes send by post, wet fixatives are avoided by status, so aerosol spray fixatives containing polyethylene glycol in ethyl alcohol were introduced (Bancroft and Stevens, 1982). The present study aimed to investigate comparative studies of dry and wet oral smear.

\section{Material and methods}

This study was carried out in saveetha dental college. Forty oral smears were collected over a period of three months from patients who visited our institution for dental problems. The smears were collected using ice cream sticks. One set of wet smears and other set which include air dried smears. Each set with 20 smears, overall 40 smears were prepared. One set of slides immediately fixed in $95 \%$ ethanol for $30 \mathrm{~min}$ and labeled Wet Fixed (WF).The other set was air-dried for 2 hours rehydrated using the honey solution prior to staining labeled Dried Fixed (DF). Then the slides were pooled and stained by standard papanicolaus method and examined by two experienced cytopathologist under the light microscope.

\section{Results}

This study revealed that immersion of air dried PAP smears in honey for 30 minutes is effectively same as a wet smear which is a routine method (Fig. 1). The interpretation of smears and the quality of staining is preserved equal or superior (figure 2 and 4). The procedure of air-dried and rehydration is simple, fast, inexpensive and highly effective. This study showed that honey can be used as a rehydrating agent which is less hazardous than chemicals. 


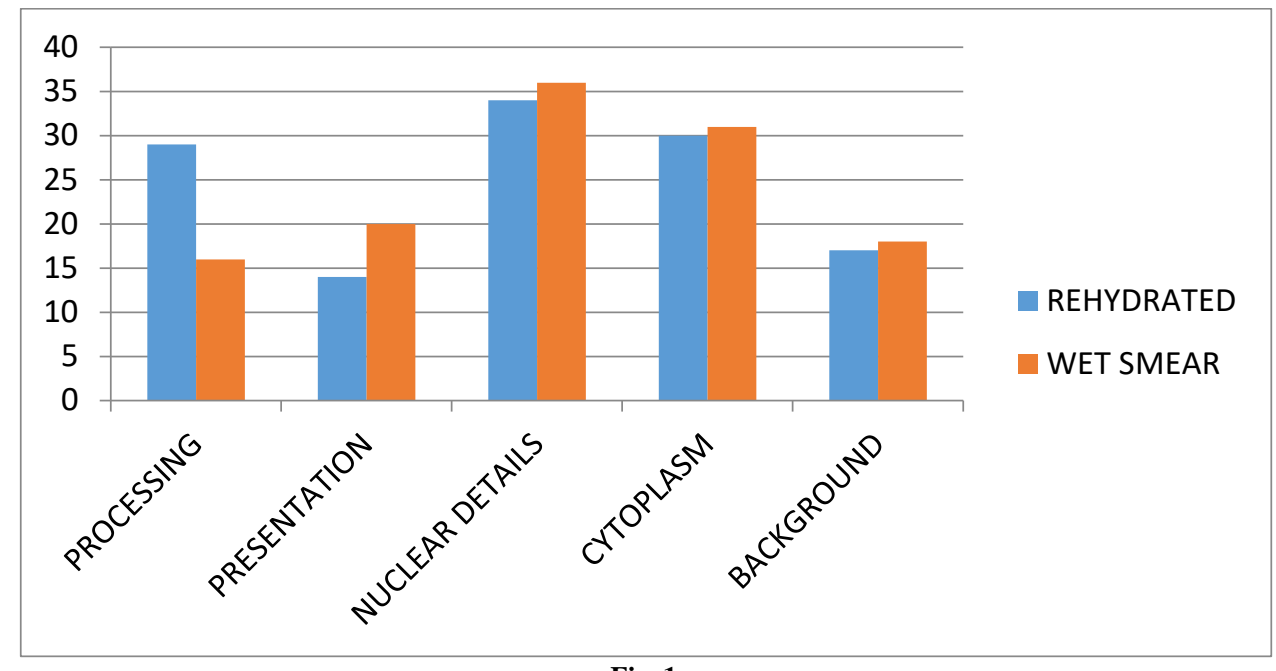

Fig. 1:

\section{Discussion}

Pap is the accurate stain for assessment of chromatin in oral smears and ensures optimal resemblance to corresponding cells in histologic sections; however, a subject is usually immediate fixation in 95\% ethanol (Chan and Kung, 1988). Rehydration of airdried pap smears within two hours of preparation produces staining quality equal or superior to that of conventional, wet fixed ones.shivarman. It has the added advantage of being less cumbersome for the clinical personnel involved in smear making. The rate of air-drying cannot be prevented around the edges of smears where diagnostic cells are populated. For this popurse it is necessary to rehydrate these dried-up cells. This study investigated that, immersion of air-dried pap smears in honey for 30 minutes, effectively but retained squamous cells present. The results of this study showed honey is equally efficient as alcohol fixed wet smears with respect to cytoplasmic staining, nuclear details. Since that study revealed superiority in cytoplasmic staining of AirDried Rehydrated Fixation (ARF) smears (Sivarman and Iyengar, 2002: Gupta et al., 2003). The procedure of air-drying and rehydration is simple, fast, inexpensive and highly effective. The rehydration time is 30 minutes because this was found to be the optimal time for clearing up the background ( $\mathrm{Ng}$ et al., 1994). This renders search for diagnostic cells less tedious and avoids the problem of overlapping red blood cells obscuring cellular details. Fig. 2 and 3: Photomicrograph showing a section of wetfixed, air dried oral smeared sample.

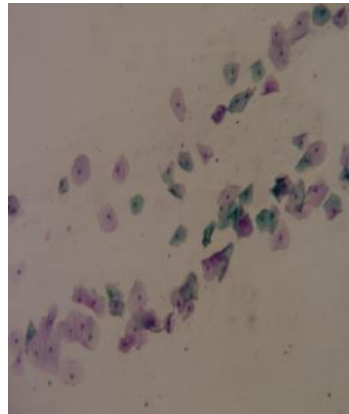

Fig. 2:

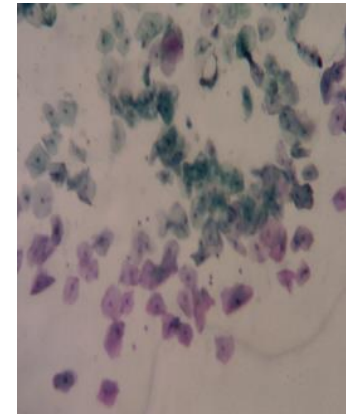

Fig. 3:
Rehydration is recommended for several other advantages as well. The smears can be spread more thinly and leisurely, the problem of falling off larger particles or thicker portions of the smear in wet fixation can be avoided, the cells have better adhesion to slides. The cells are flatter and the depth of focus on nuclei is much shallower, which is a great advantage in taking photomicrographs (Chan and Kung, 1988). In this study, we use honey as the rehydrated fuid; however, the result is as similar as that of the alcohol fixation.

\section{Conclusion}

From this study, we conclude that rehydrated honey solution can be used as a substitute for usual fixation done with alcohol in PAP smears.

\section{References}

[1] Chan JKC, Kund ITM. Rehydration of air dried smears with normal saline: Application in fine needle aspiration cytologic examination. Am J Clin Pathol 1988; 89:30-4. https://doi.org/10.1093/ajcp/89.1.30.

[2] Ng WF, Choi FB, Chund LLH, Wu C, Lung CF, Ng CS. Rehydration of air dried smears with normal saline: Application in fluid cytology. Acta Cytol 1994; 38:56-64.

[3] Dahlstrom JE, Holdsworth J, Beassett ML, Jain S. Rehydaration of air dried smears: An alternative method for cytologic analysis of exfoliative cells. Acta Cytol 1999; 43:214-7. https://doi.org/10.1159/000330979.

[4] Lencioni LJ, Staffiuri JJ, Cardonnet LJ. Vaginal and urinary sediment smear staining technique without previous fixation: Adapted to Papanicolaou's and Shorr's methods. J Lab Clin Med 1954; 44:595-9.

[5] Bales CE, Durfee GR. Cytologic techniques: Part I. In: Koss LG, ed Diagnostic cytology and its histologic basis. 4th ed. Philadelphia: Lippincott, 1992:1451-3. 